A one-to-one mapping of the points, $P$, of the surface, $S$, on the points, $Q=Q_{P}$, of another surface, $T$, is called isometric if, by virtue of the mapping, the squared lineelement, $d s^{2}$, on $S$ (i.e., the so-called "metric" or "first fundamental form", $g_{i k} d u^{i} d u^{k}$, on $S$ ) coincides with the squared line-element on $T$.

In order that this be the case, it is necessary that the Gaussian curvature of $S$ at any point $P$ be identical with the Gaussian curvature of $T$ at the corresponding point $Q=Q_{P}$. This necessary condition is sufficient as well in the particular case of surfaces of constant Gaussian curvature. Since cylinders are of constant Gaussian curvature, the constant being 0 , this embeds the trivial example (3) into the general theory.

\title{
FREE LONGITUDINAL VIBRATION OF A PROLATE ELLIPSOID, CLAMPED CENTRALLY*
}

By JAMES S. KOUVELITES (Sloane Physics Laboratory, Yale University**)

Introduction. It was originally shown by Poisson ${ }^{1}$ that the magnetization is uniform and parallel throughout the interior of an ellipsoid of magnetically isotropic material placed in a previously uniform and parallel static field. Since this unique property of the ellipsoid allows an exact calculation of its demagnetizing factor as well as a simple field analysis, the prolate spheroid was chosen as the appropriate shape for the specimens whose magnetostrictive vibrational properties are being studied in this laboratory. ${ }^{2}$ Although the magnetostrictive vibration is forced rather than free, the damping factor has been found to be so small for the materials tested, that through an investigation of the free vibration, a reasonably good approximation to some of the resonance phenomena may be obtained.

Analysis and discussion. For later comparison with the corresponding expressions for the ellipsoid, the differential equation of motion, its integrated solution, and a subsidiary expression for the frequencies of resonance for free longitudinal vibration of a bar of constant cross-section, clamped centrally, ${ }^{3}$ are

$$
\begin{gathered}
\frac{\partial^{2} \xi}{\partial x^{2}}=\frac{\rho}{k} \frac{\partial^{2} \xi}{\partial t^{2}} \\
\xi=A \sin \left(\omega\left(\frac{\rho}{k}\right)^{1 / 2} x\right) \sin (\omega t+\phi) \\
f_{R_{n}}=\frac{n}{4 a}\left(\frac{k}{\rho}\right)^{1 / 2}, \quad n=1,3,5, \cdots
\end{gathered}
$$

*Received Sept. 26, 1950.

**Assisted by the Office of Naval Research.

${ }^{1}$ As described in J. C. Maxwell, A treatise on electricity and magnetism, vol. II, 3rd ed., Clarendon Press, Oxford, 1892, pp. 66-69.

${ }^{2}$ J. S. Kouvelites and L. W. McKeehan, Magnetostrictive vibration of prolate spheroids. Preliminary measurements, Rev. Sci. Instr., in press.

${ }^{3}$ Rayleigh, Theory of sound, vol. I, \$150. 
where $x$ is the coordinate along the longitudinal axis (the origin being at the center of the bar), $t$ the time, $\xi$ the longitudinal displacement, $\rho$ the volume density, $k$ the coefficient of elasticity, $a$ the half-length of the bar, $\omega$ the angular frequency, $A$ and $\phi$ arbitrary amplitude and phase constants.

For an elastically-isotropic prolate ellipsoid with two eccentricities nearly equal to unity as in the physical problem of magnetostrictive vibration, it appears reasonable to assume that the equiphase surfaces of $\xi$ are planes perpendicular to the major axis. ${ }^{4}$ Applying the second law of motion to a transverse plane lamina of thickness $d x$ of an ellipsoid bounded by the surface

$$
\begin{gathered}
\frac{x^{2}}{a^{2}}+\frac{y^{2}}{b^{2}}+\frac{z^{2}}{c^{2}}=1, \quad \text { where } 2 a \text { is the major axis, } \\
\frac{\partial F}{\partial x} d x=\frac{\partial^{2} \xi}{\partial t^{2}} d m, \quad \text { where } d m=\pi y z \rho d x=\pi b c\left(1-\frac{x^{2}}{a^{2}}\right) \rho d x .
\end{gathered}
$$

Since Hooke's law may be stated as

$$
F=\pi b c\left(1-\frac{x^{2}}{a^{2}}\right) k \frac{\partial \xi}{\partial x},
$$

the equation of motion becomes

$$
\frac{\partial^{2} \xi}{\partial x^{2}}-\frac{2 x}{a^{2}-x^{2}} \frac{\partial \xi}{\partial x}=\frac{\rho}{k} \frac{\partial^{2} \xi}{\partial t^{2}},
$$

which is a definite departure from the familiar wave equation (1) appropriate to a channel of uniform cross section.

The separation of the variables, $x$ and $t$, is accomplished as usual by letting $\xi=$ $X(x) T(t)$, which is substituted into equation (4). Both sides of the resultant expression may then be set equal to the constant, $-\omega^{2} \rho / k$, yielding:

$$
\begin{gathered}
\frac{d^{2} T}{d t^{2}}+\omega^{2} T=0 \\
\frac{d^{2} X}{d x^{2}}-\frac{2 x}{a^{2}-x^{2}} \frac{d X}{d x}+\omega^{2} \frac{\rho}{k} X=0
\end{gathered}
$$

Thus, $\xi=X \sin (\omega t+\phi)$ where $X$ may be obtained as an explicit function of $x$ by inserting it into Eq. (6) in the form of the power series,

$$
X=A_{0}\left(\frac{x}{a}\right)^{p}+A_{1}\left(\frac{x}{a}\right)^{p+1}+\cdots+A_{s}\left(\frac{x}{a}\right)^{p+s}+\cdots
$$

As a result, it is found that, if $p=0$, both $A_{0}$ and $A_{1}$ have arbitrary, finite values and the coefficients of Eq. (7) satisfy the condition

$$
A_{s+2}=\frac{s}{s+2} A_{s}+\frac{N}{(s+1)(s+2)}\left(A_{s-2}-A_{s}\right),
$$

where $N=\omega^{2} a^{2} \rho / k$

${ }^{4}$ See Rayleigh ${ }^{3}$, Vol. II, \$265 and P. M. Morse, Vibration and sound, McGraw-Hill Book Co., New York, 1936, pp. 216-217 for analogous treatment of sound transmission in tubes of varying cross-section. 
Moreover, since the physical situation requires that the ellipsoid be clamped centrally (i.e. $\xi=0$ at $x=0$ at any time), $A_{0}, A_{2}, A_{4}$, etc., must be zero. The series solution for $X$, subject to Eq. (8), then becomes

$$
X=A_{1}\left(\frac{x}{a}\right)+A_{3}\left(\frac{x}{a}\right)^{3}+A_{5}\left(\frac{x}{a}\right)^{5}+\cdots .
$$

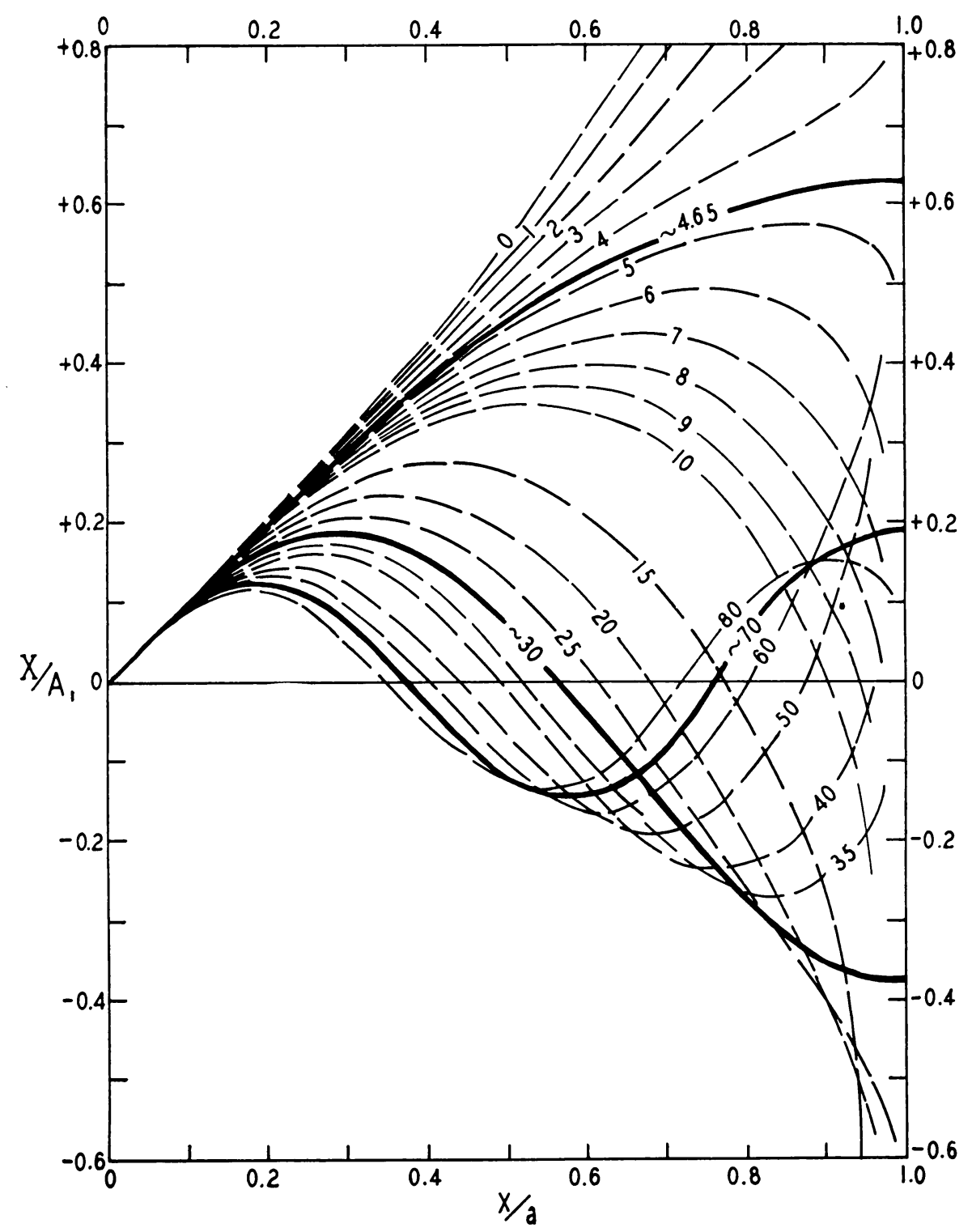

Fig. 1.

There is no force exerted on the ends of the ellipsoid. Consequently, the second boundary condition of the problem is that $d X / d x=0$ at $x= \pm a$. The particular values 
of $N$ which satisfy this condition, are found by a trial and error process from Eqs. (8) and (9). It is interesting to note that Eq. (9), for $x= \pm a$, is divergent for all values of $N$ except those corresponding to the modes of vibration satisfying the second boundary condition.

In Fig. $1, X / A_{1}$, has been plotted as a function of $x / a$ for various values of $N$, the thickly-drawn curves representing the "standing-wave" patterns for the fundamental mode and its first two overtones. It was sufficient to show the deflection variations over only one half the length of the ellipsoid because there is symmetry about the plane $x=0$.

The frequencies of resonance for the ellipsoid may thus be expressed as

$$
f_{R_{n}}=\frac{n}{4 a}\left(\frac{k}{\rho}\right)^{1 / 2} \quad \text { where } n \simeq 1.38,3.49,5.33, \cdots,
$$

indicating the inharmonic nature of the overtones. However, a comparison between Eqs. (3) and (10) reveals that the consecutive overtones of the vibrating ellipsoid have ratios closer and closer to the ratios of adjacent odd integers as the value of $n$ increases.

Acknowledgements. I wish to thank Professor L. W. McKeehan of this laboratory, whom I am assisting in the study of magnetostrictive vibration, and Professor F. J. Beck, Jr., of the Dunham Laboratory of Electrical Engineering, for encouraging discussions of this problem.

\section{THE LEAST SQUARES SOLUTION FOR A SET OF COMPLEX LINEAR EQUATIONS*}

By R. TURETSKY (Aberdeen Proving Ground)

Consider the set of $m$ observational equations whose matrix representation is

$$
A z \sim w,
$$

where $A$ is an $m \times n(m \geq n)$ matrix of rank $n$ whose elements are prescribed complex quantities, while $w$ is an $m \times 1$ matrix. We seek that $z$ (an $n \times 1$ matrix) which minimizes the sum of the squares of the absolute values of the components of the vector $A z-w$.

Set $A=B+i C, z=x+i y, w=u+i v$, where $B, C, x, y, u$, and $v$ are real matrices. Then Eq. (1) is equivalent to

$$
\left(\begin{array}{rr}
B & -C \\
C & B
\end{array}\right)\left(\begin{array}{l}
x \\
y
\end{array}\right) \sim\left(\begin{array}{l}
u \\
v
\end{array}\right)
$$

This is the matrix representation for a set of real observational equations. To obtain the normal equations, ${ }^{1}$ we multiply on the left by the transpose of the coefficient matrix

${ }^{*}$ Received July 28, 1950.

'See Whittaker and Robinson, Calculus of observations, Chapter IX. 